\title{
A Geometric Method of Singularity Avoidance for Kinematically Redundant Planar Parallel Robots
}

\author{
Nicholas Baron, Andrew Philippides and Nicolas Rojas
}

\begin{abstract}
Methods for avoiding singularities of closed-loop robot mechanisms have been traditionally based on the value of the determinant or the condition number of the Jacobian. A major drawback of these standard techniques is that the closeness of a robot configuration to a singularity lacks geometric, physical interpretation, thus implying that it is uncertain how changes in the robot pose actually move further away the mechanism from such a problematic configuration. This paper presents a geometric approach of singularity avoidance for kinematically redundant planar parallel robots that eliminates the disadvantages of Jacobian-based techniques. The proposed method, which is based on the properties of instantaneous centres of rotation, defines a mathematical distance to a singularity and provides a reliable way of moving the robot further from a singular configuration without changing the pose of the end-effector. The approach is demonstrated on an example robot mechanism and the reciprocal of the condition number of the Jacobian is used to show its advantages.
\end{abstract}

\section{Introduction}

A parallel robot is a closed-loop kinematic chain mechanism where the end-effector is connected to the base via two or more independent kinematic chains. A key feature of parallel robots is that once the actuated joints are locked, the mechanism becomes, in general, rigid. However, it is well known that there are particular configurations, known as singularities, in which the total number of degrees of freedom changes instantaneously and consequently the robot loses its rigidity. Herein, the term singularity will refer to the so called forward kinematics singularity, which

Nicholas Baron, Andrew Philippides

University of Sussex, Brighton BN1 9RH, UK. e-mail: \{n.baron, andrewop\}@ sussex.ac.uk

Nicolas Rojas

Imperial College London, London SW7 1NA, UK. e-mail: n.rojas@imperial.ac.uk 
describes the instance where different branches of the direct position problem meet [1]. Singular configurations are problematic because the task wrench cannot be resisted by active joint torques, or equivalently the end-effector is able to move despite the input velocities of the active joints being zero [2]. In fact, these problems are not just confined to the exact point at which the robot is in a singularity; the robot can exhibit such behavior when close enough to a singular configuration. This is a major issue in terms of the control of the robot and also for robot safety.

The traditional method of singularity analysis is to calculate the Jacobian of the robot, that is, the matrix that describes the relationship between the input velocities of the actuated joints and the output velocity of the end-effector, and then to compute an index, such as the reciprocal of the condition number or the determinant [3]. The point at which the robot is in a singularity is given by the instance when the value of this index is zero. The problem with using Jacobian indexes as means for performing the singularity analysis is that they have no straightforward geometric interpretation; meaning that for any non-zero value, it is not clear how close the robot is to a singular configuration. Resultantly, by just computing the value of such indexes, there is no physical understanding of the robot's proximity to a singularity and how to move away from it. Many methods of singularity avoidance have been proposed that are based on the calculation of Jacobian indexes [4], [5].

In this paper, a simple, geometric method of singularity avoidance for kinematically redundant planar parallel robots is proposed. Kinematically redundant parallel robots are architectures with more actuators than required by the task workspace, but which all contribute to make the robot rigid when locked without serially connected subsets [6,7]. The suggested method, which is based on the properties of instantaneous centres of rotation, has a direct geometric interpretation, providing a physical insight of the robot's proximity to a singularity. These characteristics make it possible to compute a new configuration of the robot for which the distance to a singularity is greater, and yet the pose of the end-effector remains the same.

The paper is structured as follows. In section 2, the concept of instantaneous centres of rotation and its use to determine singularities of planar parallel manipulators is discussed. In section 3, the geometric method of singularity avoidance is proposed which involves, firstly, computing the robot's proximity to a singularity using the properties of instantaneous centres of rotation and, secondly, manipulating its configuration for moving further away from it. The method is demonstrated numerically on an example robot mechanism in section 4, in which the reciprocal of the condition number of the Jacobian is also calculated for comparison. Finally, we conclude in section 5 .

\section{Instantaneous Centres of Rotation and Singularities}

The Instantaneous Centre of Rotation (ICR) between two rigid bodies that are moving relative to one another in the same plane is defined as the instantaneous location of a coincident pair of points of the two bodies of which the absolute velocities are 
equal [8]. This concept can be applied to any kinematic chain with mobility one in order to find the ICR between any two of the links in the system. Following this, the singularity analysis can be carried out on a planar parallel robot, that in general has a mobility zero when the actuators are locked, by considering the robot as a composition of its mobility one sub-mechanisms; these are the kinematic chains of mobility one within the robot that are obtained by removing one or more of the the links that connect the base to the platform. The mobility one sub-mechanisms of a parallel robot can be identified by using, for instance, the extended ChebychevKutzbach-Grübler formula [9]

$$
M=F-\sum_{i=1}^{\lambda} t_{i}
$$

where $\lambda=J-L+1$ is the number of independent loops in the kinematic chain, $t_{i}$ is the motion type of the $i^{\text {th }}$ independent loop ( $t_{i}=3$ in the planar case), $J$ is the total number of joints, $L$ is the number of links, and $F$ is the total number of degrees of freedom of the joints.

A singularity in a planar parallel robot is produced in the instance where the ICR between the platform and the base of each mobility 1 sub-mechanism coincide, since in these configurations the platform is able to, instantaneously, rotate about such a point [7]. It therefore follows that a robot's proximity to a singular configuration can be determined by calculating the position of the ICRs between the platform and the base for each of these sub-mechanisms and computing the content of the simplex formed by them-i.e., length in the case of two points. It can be shown that for planar parallel robots of up to three independent loops, the geometric conditions that cause two of these ICRs to coincide are the same that cause any pairing of these ICRs to coincide, hence it is only necessary to calculate the ICR between the platform and the base for two of the mobility 1 sub-mechanisms. Additionally, if the vector between these ICRs is found, the robot can be reconfigured such that the ICRs are moved further apart from each other, thus moving the robot further away from a singularity.

\section{Geometric Method of Singularity Avoidance}

The aim of the method is to calculate the vector between the ICRs between the base and the platform of two of the mobility 1 sub-mechanisms of the kinematically redundant planar parallel robot, and then subsequently reconfigure the robot such that one of these ICRs moves further away from the other. The advantage of kinematically redundant mechanisms is that it is possible to reconfigure at least one of the legs without manipulating the pose of the end-effector. This means that it is possible to change the positions of one of the ICRs between the platform and the base of one of the mobility 1 sub-mechanisms without manipulating the pose of the end-effector. Therefore, if the vector between this ICR and another ICR (one that 


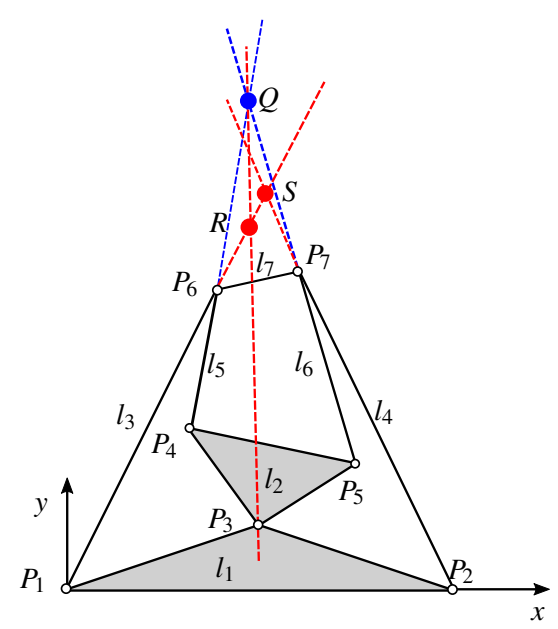

Fig. 1 A kinematic diagram of the kinematically redundant planar parallel robot proposed in [7] with RPR legs. $R$ and $S$ are the ICR(1,7) for the sub-mechanisms where links 4 and 5 are removed, respectively.
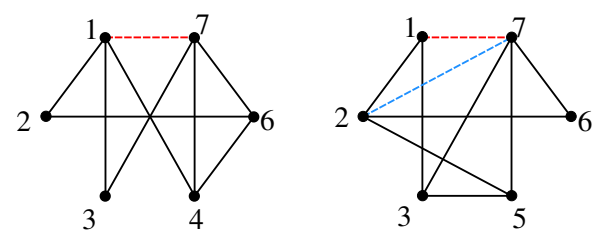

Fig. 2 Circle diagrams for the $M=1$ sub-mechanisms with link 5 removed (left) and link 4 removed (right).

is independent of the configuration of the redundant leg) is known, then the former ICR can be repositioned such that it moves further away from the latter ICR, and thus the robot moves further away from a singularity.

This is best understood by examining an example robot mechanism; herein, the kinematically redundant planar parallel robot proposed in [7] is used, in this case with revolute-prismatic-revolute (RPR) legs. A diagram of this robot is shown in Fig. 1; it consists of a platform, a base, four RPR legs and an additional ternary link that is referred to as the moving base; the underline in this convention means that the corresponding joint is actuated. The moving base is joined to the base via a passive revolute joint and to the platform via two of the legs, the other two legs connect the platform to the base.

Firstly, the ICRs between the platform and the base for the mobility 1 submechanisms of the robot need to be calculated. Herein, the ICR between two links i and $\mathrm{j}$ will be denoted by $\operatorname{ICR}(\mathrm{i}, \mathrm{j})$; for example, according to the notation of Fig. 1, the ICR between the base, link 1, and the platform, link 7, for each of the mobility 1 sub-mechanisms of this robot is denoted by $\operatorname{ICR}(1,7)$. In order to determine the position of $\operatorname{ICR}(1,7)$ for each sub-mechanism, a book-keeping system through the 
use of so-called Circle Diagrams is used [10]. The links of the mechanism are denoted by number and a filled line between two links is drawn if the ICR between the two links is known-i.e., the links share a joint. A dotted line is drawn between two links to indicate a desired, unknown ICR; it is possible to find the position of this ICR if the dotted line is a common side of two triangles of which the other sides are filled lines.

In the analyzed robot, five sub-mechanisms which have mobility 1 can be identified. However, it is only necessary to calculate the ICRs between the platform and the base of two of these sub-mechanisms as the resulting conditions are equivalent; these mechanisms are that where Link 5 is removed, which will be referred to as SM-A, and that where Link 4 is removed, which will be referred to as SM-B. The circle diagrams for SM-A and SM-B are displayed in Fig. 2.

For SM-A, it can be seen by inspecting the circle diagram that the ICR between the platform and the base, $\operatorname{ICR}(1,7)$, point $\mathbf{S}$ in Fig. 1, is positioned at the point of intersection between the lines which pass along links 3 and 4. For SM-B, the determination of ICR $(1,7)$ is as follows. In the circle diagram, the dotted line drawn between links 1 and 7 is not the common side of two otherwise known triangles therefore an additional unknown ICR must be found first, this is $\operatorname{ICR}(2,7)$ and its position is located at the point of intersection between the lines that pass along Links 5 and 6, point $\mathbf{Q}$ in Fig. 1. ICR $(1,7)$ can then be found by drawing two lines; one which passes through $Q$ and $P_{3}$, and another which passes along link 3. The point at which these lines intersect is the position of $\operatorname{ICR}(1,7)$ for SM-B, this is point $\mathbf{R}$ in Fig. 1.

\subsection{Moving away from a singular configuration}

In what follows, the horizontal and vertical components of a point $\mathbf{P}_{i}$ in a given reference frame are denoted by $x_{i}$ and $y_{i}$, and the gradient of the line which passes through points $\mathbf{P}_{i}$ and $\mathbf{P}_{j}$ is denoted by $m_{i, j}$. Then, from a robot configuration, the coordinates of $\mathbf{R}, \mathbf{Q}$, and $\mathbf{S}$ can be computed as

$$
\begin{aligned}
& \mathbf{S}=\left[\begin{array}{c}
\frac{1}{m_{1,6}-m_{2,7}}\left(y_{2}-m_{2,7} x_{2}-y_{1}+m_{1,6} x_{1}\right) \\
\frac{m_{1,6}}{m_{1,6}-m_{2,7}}\left(y_{2}-m_{2,7} x_{2}-y_{1}+m_{1,6} x_{1}\right)+y_{1}-m_{1,6} x_{1}
\end{array}\right], \\
& \mathbf{Q}=\left[\begin{array}{c}
\frac{1}{m_{4,6}-m_{5,7}}\left(y_{5}-m_{5,7} x_{5}-y_{4}+m_{4,6} x_{4}\right) \\
\frac{m_{4,6}}{m_{4,6}-m_{5,7}}\left(y_{5}-m_{5,7} x_{5}-y_{4}+m_{4,6} x_{4}\right)+y_{4}-m_{4,6} x_{4}
\end{array}\right], \\
& \mathbf{R}=\left[\begin{array}{c}
\frac{1}{m_{1,6}-m_{3, Q}}\left(y_{3}-m_{3, Q} x_{3}-y_{1}+m_{1,6} x_{1}\right) \\
\frac{m_{1,6}}{m_{1,6}-m_{3, Q}}\left(y_{3}-m_{3, Q} x_{3}-y_{1}+m_{1,6} x_{1}\right)+y_{1}-m_{1,6} x_{1}
\end{array}\right] .
\end{aligned}
$$

It can be seen by inspecting the mechanism that the pose of the platform must necessarily change if the length of either link 3 or link 4 is modified. However, changing the lengths of link 5 and link 6 does not necessarily alter the pose of link 
7. This means that point $\mathbf{R}$ is the ICR whose position can be altered in order to avoid singular configurations. Let $\mathbf{d}$ be the vector from $\mathbf{S}$ to $\mathbf{R}$. Hence, the robot can be moved further from a singular configuration by moving the position of $\mathbf{R}$ along the direction of this vector; this can be achieved by multiplying $\mathbf{d}$ by a scalar $\lambda>1$, that is,

$$
\mathbf{R}^{*}=\mathbf{S}+\lambda \mathbf{d}
$$

where $\mathbf{R}^{*}$ is the new position of $\mathbf{R}$.

Having computed $\mathbf{R}^{*}$, the new position of $\mathbf{Q}$, say $\mathbf{Q}^{*}$, needs to be determined. The only requirement of $\mathbf{Q}^{*}$ is that it has to lie on the line which passes through $\mathbf{R}^{*}$ and $\mathbf{P}_{3}$. Its position is determined by calculating the equation of this line and then selecting a feasible gradient of link $5, m_{4,6}$. The aim of the method therefore is to obtain a value for $m_{4,6}$ that produces the desired value of $\mathbf{R}^{*}$. The first step is to calculate an expression of $\mathbf{P}_{4}$ in terms of $m_{4,6}$. $\mathbf{P}_{4}$ is positioned at one of the points of intersection between the line of gradient $m_{4,6}$ that passes through the centre of $\mathbf{P}_{6}$ and the circle whose centre is that of $\mathbf{P}_{3}$ and whose radius is the distance between $\mathbf{P}_{3}$ and $\mathbf{P}_{4}, d_{3,4}$. Thus,

$$
\mathbf{P}_{4}=\left[\begin{array}{c}
\frac{-b \pm \sqrt{b^{2}-4 a c}}{2 a} \\
m_{4,6} \frac{-b \pm \sqrt{b^{2}-4 a c}}{2 a}+y_{6}-m_{4,6} x_{6}
\end{array}\right],
$$

where $a=1+m_{4,6}^{2}, b=2 m_{4,6}\left(y_{6}-m_{4,6} x_{6}-y_{3}\right)-2 x_{3}$, and $c=x_{3}^{2}+\left(y_{6}-m_{4,6} x_{6}-\right.$ $\left.y_{3}\right)^{2}-d_{3,4}^{2}$. The \pm signs in equation (6) denote the two possible positions of $\mathbf{P}_{4}$, either are feasible however the length of link 5 must lie within the limits of the actuated prismatic joint.

The positions of the centres of the remaining unknown joints are found by solving the inverse kinematics problem-a method to do this is discussed in [7]. Then, with an expression for the position of $\mathbf{P}_{5}$ obtained, the gradient of link $6, m_{5,7}$, is calculated in terms of $m_{4,6}$. Now, a value of $m_{4,6}$ is calculated such that the lines which pass through links 5 and 6 intersect at a point which lies on the line which passes through $\mathbf{P}_{3}$ and $\mathbf{R}^{*}$. Then,

$$
\mathbf{Q}^{*}=\left[\begin{array}{c}
\frac{c_{5,7}-c_{4,6}}{m_{4,6}-m_{5,7}} \\
m_{4,6} \frac{c_{5,7}-c_{4,6}}{m_{4,6}-m_{5,7}}+c_{4,6}
\end{array}\right]
$$

where $c_{4,6}=y_{6}-m_{4,6} x_{6}$ and $c_{5,7}=y_{7}-m_{5,7} x_{7}$. Finally, the components of $\mathbf{Q}^{*}$ in terms of $m_{4,6}$ can be expressed as

$$
y_{Q^{*}}-m_{3, R^{*}} x_{Q^{*}}-c_{3, R^{*}}=0,
$$

where, $c_{3, R^{*}}=y_{3}-m_{3, R^{*}} x_{3, R^{*}}$ is the y-intercept of the line which passes through $\mathbf{P}_{3}$ and $\mathbf{R}^{*}$.

Equation (8) is a radical equation in $m_{4,6}$, the roots of which are the possible values of $m_{4,6}$. Since only one solution is required, root-finding algorithms, such as the 

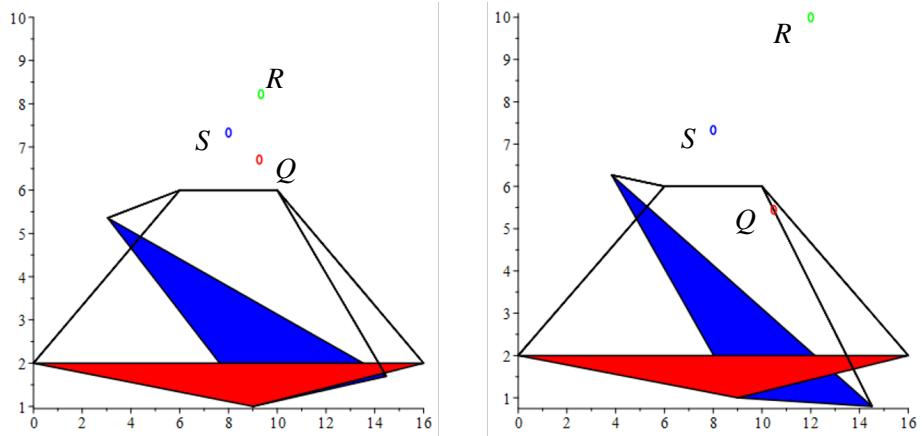

Fig. 3 Original and improved configurations of the robot (left and right, respectively).

Newton-Raphson method, can be used. The positions of $\mathbf{P}_{4}$ and $\mathbf{Q}^{*}$ are then found by substituting the obtained value back into equations (6) and (7), respectively.

\section{Numerical Example}

In this section the method is carried out on an example mechanism with the same architecture that is examined in the previous section. Firstly, $\mathbf{d}$ is calculated for the mechanism with the following joint coordinates: $\mathbf{P}_{1}=(0,2)^{T}, \mathbf{P}_{2}=(16,2)^{T}, \mathbf{P}_{3}=$ $(9,1)^{T}, \mathbf{P}_{4}=(3.05,5.36)^{T}, \mathbf{P}_{5}=(14.47,1.70)^{T}, \mathbf{P}_{6}=(6,6)^{T}$ and $\mathbf{P}_{7}=(10,6)^{T}$. Using these values, and by computing the necessary gradients, the position of $\mathbf{S}, \mathbf{Q}$ and $\mathbf{R}$ is found using equations (2)-(4) and, resultantly, $\mathbf{d}$ is obtained by subtracting $\mathbf{S}$ from $\mathbf{R}$. Hence, $\mathbf{S}=(8,7.33)^{T}, \mathbf{Q}=(9.26,6.71)^{T}, \mathbf{R}=(9.33,8.22)^{T}$ and $\mathbf{d}=$ $(1.33,0.89)^{T}$. This configuration is displayed on the left hand side of Fig. 3. $\mathbf{R}^{*}$, the new position of $\mathbf{R}$, is then calculated with equation (5), for instance $\mathbf{R}=(12,10)^{T}$ when $\lambda=3$, subsequently, using equations (6)-(8), a radical equation in terms of $m_{4,6}$ is obtained. A feasible solution of such an equation is $m_{4,6}=-0.124$.

Given the above value for $m_{4,6}$, the new position of $\mathbf{Q}$ is then $\mathbf{Q}^{*}=(10.48,5.44)^{T}$ and consequently $\mathbf{d}=(4,2.67)^{T}$. The positions of the centres of joints $\mathbf{P}_{1}, \mathbf{P}_{2}, \mathbf{P}_{3}$, $\mathbf{P}_{6}$ and $\mathbf{P}_{7}$ are unchanged, and the remaining joints are recalculated. In this example, the kinematic redundancy of the robot is utilised as the pose of the end-effector is not modified. This configuration is displayed on the right hand side of Fig. 3.

The advantage of the proposed method of singularity avoidance is that it provides a straightforward interpretation of the robot's proximity to a singularity. The magnitude of $\mathbf{d}$ can be compared with the dimensions of the robot, or its resolution, such that there is a clear context to the size of this value. On the other hand, if we perform the above analysis using a traditional Jacobian-based index, such as the reciprocal of the condition number, we obtain a value of $1.094 \times 10^{-2}$ for the original configuration and $2.118 \times 10^{-2}$ for the second, improved configuration. It can be 
seen that these values provide no real insight into how close physically the robot is to a singularity.

\section{Conclusion}

A main problem with traditional methods of singularity analysis based on indexes of the Jacobian matrix, such as the reciprocal of the condition number, is the absence of a straightforward geometric interpretation, implying that, in essence, they are meaningless for all non-zero values. In this paper, a geometric method of singularity avoidance for kinematically redundant planar parallel robots, based on the properties of instantaneous centres of rotation, is introduced; the technique provides a physical understanding of the robot's proximity to a singularity and allows the definition of escaping trajectories that effectively deviate the robot from dangerous configurations. These characteristics, which are simple to implement, open the door to further research in geometric planners of singularity-free paths for closed-loop manipulators.

\section{References}

1. C. Gosselin and J. Angeles, "Singularity analysis of closed-loop kinematic chains," IEEE Transactions on Robotics and Automation, vol. 6, no. 3, pp. 281-290, 1990.

2. J. F. O'Brien and J. T. Wen, "Kinematic control of parallel robots in the presence of unstable singularities," in IEEE Transactions on Robotics and Automation, vol. 1. IEEE, 2001, pp. 354-359.

3. J.-P. Merlet, "Jacobian, manipulability, condition number, and accuracy of parallel robots," Journal of Mechanical Design, vol. 128, no. 1, pp. 199-206, 2006.

4. S.-H. Cha, T. A. Lasky, and S. A. Velinsky, "Singularity avoidance for the 3-rrr mechanism using kinematic redundancy," in IEEE International Conference on Robotics and Automation. IEEE, 2007, pp. 1195-1200.

5. S. Bhattacharya, H. Hatwal, and A. Ghosh, "Comparison of an exact and an approximate method of singularity avoidance in platform type parallel manipulators," Mechanism and Machine Theory, vol. 33, no. 7, pp. 965-974, 1998.

6. C. Gosselin, T. Laliberté, and A. Veillette, "Singularity-free kinematically redundant planar parallel mechanisms with unlimited rotational capability," IEEE Transactions on Robotics, vol. 31, no. 2, pp. 457-467, 2015.

7. N. Baron, A. Philippides, and N. Rojas, "A generic kinematically redundant planar parallel robot with full rotatability," Under Review.

8. H. M. Daniali, "Instantaneous center of rotation and singularities of planar parallel manipulators," International Journal of Mechanical Engineering Education, vol. 33, no. 3, pp. 251-259, 2005.

9. N. Rojas and A. M. Dollar, "Gross motion analysis of fingertip-based within-hand manipulation," IEEE Transactions on Robotics, vol. 32, no. 4, pp. 1009-1016, 2016.

10. R. S. Hartenberg and J. Denavit, Kinematic synthesis of linkages. New York, NY: McGrawHill, 1964, ch. 4 